\title{
Probiotics for Health Benefits: The Regulatory Concerns and Suggestive Roadmap
}

\author{
Ashish Baldi* \\ Department of Pharmaceutical Sciences and Technology, Maharaja Ranjit Singh Punjab Technical University, Bathinda (Punjab), India \\ Received: September 16, 2017; Published: October 03,2017 \\ *Corresponding author: Ashish Baldi, Professor and Head, Department of Pharmaceutical Sciences and Technology, Maharaja Ranjit Singh Punjab \\ Technical University, Bathinda, Punjab, India; Tel: +918968423848; Email: baldiashish@gmail.com
}

\begin{abstract}
Probiotics, the friendly bugs, has gained impressive attention worldwide due to their nutraceutical and pharmaceutical benefits, established in recent years. However existing regulatory regime in various countries has ambiguities regarding its classification based on intended use and respective approval process. Author and his coworkers have proposed a revised definition and categorization of probiotic products along with possible path to develop a comprehensive guideline for global enactment.
\end{abstract}

Keywords: Food; Dietary supplements; Health claims; Nutraceuticals; Probiotics; Pharmaceuticals; Regulatory guidelines.

\section{Introduction}

Probiotics, as defined by FAO/WHO, are live microorganisms, which when administered in adequate amounts confer a health benefit on the host. In recent days, use of probiotics for health benefits has increased due to their wide range of medicinal values complemented with low cost and insignificant side effects. This has resulted in commercial exploration of probiotics globally for nutraceutical and pharmaceutical applications. The major driving force behind the success of these products is growing interest in the concept of holistic health through diet regulation as preventive step to avoid various diseases. In last two decades, probiotics are also reported to be useful in treatment of some serious clinical disorders such as cancer, diabetes, cardiovascular diseases, bacterial vaginosis, urinary tract infections, allergic diseases (atopic dermatitis, allergic rhinitis) and prevention of dental caries, respiratory infections, lactose intolerance, variety of gastrointestinal disorders like diarrhea, irritable bowel syndrome etc. Recent findings have established that some of the probiotic strains may have therapeutic potential and involvement in new therapeutic pathways in dysbiosis related diseases.

The frequent and sometimes irrational use of antibiotics has also made probiotics as almost essential component of prescription specially to maintain the friendly microbial flora in gut and intestines. Ever increasing incidences of gastrointestinal diseases, market acceptance and emerging biotechnological advancements in formulation designing also resulted in expansion of probiotic market across the globe. The probiotic market is presently growing at a CAGR of $6.8 \%$ with an expected market value of USD 45 billion in 2018 attributed to food, beverage, dietary supplements and pharmaceuticals.

\section{Current Status of Probiotics}

Despite of tremendous growth in the probiotic market across the globe, till date, their development, regulatory aspects, legislative issues and technological obstacles are complex, risky and expensive enough. Regulatory agencies of different countries are regulating them under variety of categories viz. dietary supplements, natural health products, medical foods, functional foods, drugs etc. as per their intended use. However there is no clear cut demarcation of probiotic based pharmaceutical and food products. Therefore an urgent attention is needed for effective regulation and approval process of these products with established scientific evidences for their therapeutic and nutritional claims.

Regulatory Framework for Probiotics: Issues of Concerns

The existing regulatory regime or probiotic product has following issues of concern:

i. Categorization and regulatory guidelines vary from country to country.

ii. No universally accepted definition and harmonized regulations.

iii. Status of probiotics as a component of food is not established on international basis. 
iv. Confusion among all stakeholders about clear distinction between nutritional and health claims.

v. Unavailability of diagnostic tools to determine the appropriate probiotics to use in a particular situation, their safety and efficacy.

vi. Approval process of probiotics is still confusing. For example, USFDA has no regulations to control probiotics with health claims. Guidelines for 'Live Bio therapeutic Products', published in 2010, is also not completely applicable logically for probiotics. On the other hand, European regulatory framework vides its 'Nutrition and Health Claims Regulations' 2007 has clear demarcation between health claims in healthy individuals and diseased patients.

\section{Future Recommendations}

Comprehensive Guidelines: Keeping existing lacunas in the prevailing guidelines into mind, all the necessary aspects should be appropriately addressed in a comprehensive manner by regulatory agencies for the safe and judicious use of probiotic based health products. The author and his coworkers has proposed unified regulatory guidelines covering all related aspects including definition of probiotics, their categorization, identification, evaluation, manufacturing labeling and approval for the marketing of these products. The developed guidelines may be adopted comprehensively as a base document for global implementation of more effective regulatory framework for probiotic products.

Adoption of Unified Definition of Probiotics: Present definition of probiotics, as given by FAO/WHO, do not emphasize on use of mix culture, viability, nutritional or health benefit and correct dosage. Hence a new definition is proposed by author and his group considering all such confines in the prevailing definition.

Suggestive Categorization of Probiotics: A new categorization, as per their intended usage and safety parameters, has been proposed to achieve adequate regulatory control. Beneficial microbes having probiotic potential must be categorized broadly, under two broad categories "Nutribiotics" i.e. probiotics which have general or any specified nutritive claim and "Pharmabiotics" i.e. probiotics as pharmaceuticals specifically having health claims include those probiotic microbes or products based on those microbes, which have general or any specified health claim against diseases [1-8].

\section{Conclusion}

Lack of properly defined regulatory framework and variation in country wise regulations is posturing a major risk on establishment and reliability of probiotic based products. To avoid the ambiguities, harmonized and holistically established norms should be enforced at global level in unified manner. This will surely increase the acceptance of probiotic products for their evidence based nutritive/ health benefits, undeviating quality along with superior safety of patients.

\section{References}

1. Arora M, Sharma S, Baldi A (2013) Comparative Insight of Regulatory Guidelines for Probiotics in USA, India and Malaysia: A Critical Review. International Journal of Biotechnology Wellness Industries 2(2): 51-64.

2. Arora M, Baldi A (2015) Comparative Account of Quality Management and Regulatory Aspects of Products with Health Claims: Existing Approaches and Future Challenges for Probiotics and Herbal Products. Applied Clinical Research, Clinical Trials and Regulatory Affairs 2(3): 123-132.

3. Arora M, Baldi A (2015) Good Manufacturing Practice (GMP) Regulations for Probiotic Based Pharmaceuticals: Current Scenario and Suggestive Recommendations. Applied Clinical Research, Clinical Trials and Regulatory Affairs 2(3): 165-175.

4. Arora M, Baldi A, Singh D (2015) Existing Regulatory Regimes for Traditional Products with Health Claims: Insights and Prospects. In: Bansal P (Ed) Potentials and Bottleneck in Clinical Trials of Herbal Drugs. Gulab Publishers, New Delhi, India, pp. 32-50.

5. Arora M, Baldi A (2015) Regulatory Categories of Probiotics Across the Globe: A Review Representing Existing and Recommended Categorization. Indian Journal of Medical Microbiology 33(5): 2-10.

6. Sharma S, Arora M, Baldi A (2013) Probiotics in India: Current Status and Future Prospects. Pharmaspire 4: 68-77.

7. Arora M, Baldi A (2017) Selective Identification and Characterization of Potential Probiotic Strains: A Review on Comprehensive Polyphasic Approach. Applied Clinical Research, Clinical Trials and Regulatory Affairs 3(1): 60-76.

8. Arora M, Baldi A (2017) Comparative Study of Regulatory Framework for Probiotics: Current Status and Future Recommendations. Applied Clinical Research, Clinical Trials and Regulatory Affairs 4(2): 140-156.

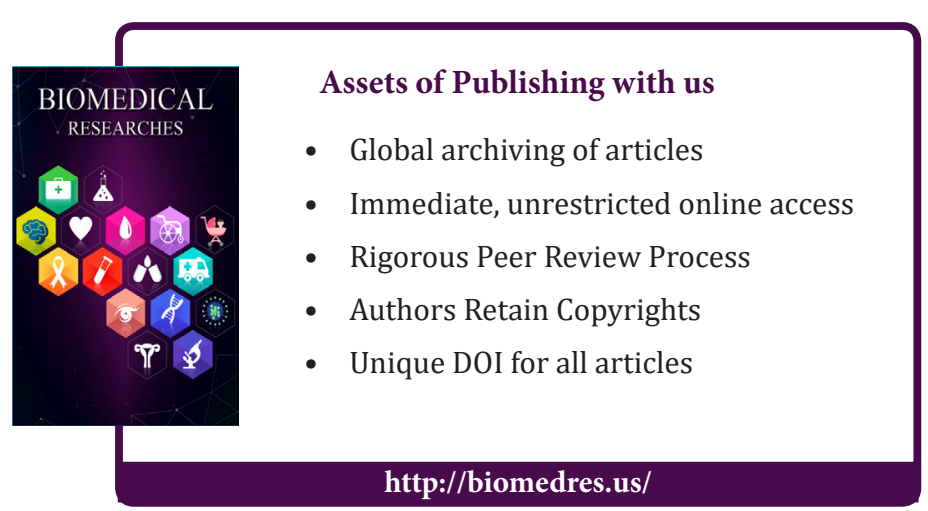

